\title{
La Guelaguetza - Una fiesta moderna de México
}

\section{Katalin Jancsó}

La fiesta es un acontecimiento casi diario e indispensable en la vida de los mexicanos. Es un buen pretexto para reunirse, festejar a un santo, comer, bailar y cantar. Octavio Paz formuló con precisión la esencia del "arte de la Fiesta" de los mexicanos:

"El arte de la Fiesta, envilecido en casi todas partes, se conserva intacto entre nosotros. En pocos lugares del mundo se puede vivir un espectáculo parecido al de las grandes fiestas religiosas de México, con sus colores violentos, agrios y puros, sus danzas, ceremonias, fuegos de artificio, trajes insólitos y la inagotable cascada de sorpresas de los frutos, dulces y objetos que se venden esos días en plazas y mercados. Nuestro calendario está poblado de fiestas."

Todos estos 'ingredientes' se reúnen en una fiesta oaxaqueña, la llamada Guelaguetza que muchas veces definen como herencia cultural de los pueblos indios de la región. El objetivo de nuestro estudio fuera de la descripción de la fiesta es presentar los elementos e influencias que podían contribuir a la creación de una tradición y demostrar que estas celebraciones llevan más bien una mezcla de signos de la época colonial y aun más del siglo XX que trae consigo el verdadero nacimiento de la fiesta actual.

\section{El origen y la historia de la Guelaguetza}

El origen de las fiestas lo atribuyen a celebraciones aztecas. En el valle de la actual Oaxaca después de los zapotecos y mixtecos a finales del siglo XV aparecieron los aztecas quienes fundaron una guarnición militar al pie de un cerro que los zapotecos llamaban "Tani Lao Nayaaloani" o "Daninayaloani" (significa Monte de Bella Vista). El lugar recibió el nombre de Huaxyacac (denominación náhuatl que significa "lugar de los guajes") que más tarde se conoció como el Cerro del Fortín (ese es el nombre actual también). Los aztecas llevaron consigo el culto a Centéotl -mejor dicho Centeociahuatl-, deidad de Maíz maduro ${ }^{3}$ que se celebraba en las faldas del cerro (teocalli de Centéotl) en el octavo mes de su calendario (hoy julio) y que consistía en cantos, bailes y músicas solicitando el favor de la diosa con el fin de lograr buenas cosechas. También eligieron y sacrificaron a una mujer que representaba a la Diosa. ${ }^{4}$ Según los organizadores las fiestas actuales se remontan hasta esos tiempos.

En 1521 llegaron los primeros españoles y fundaron la Villa de Antequera que obtuvo privilegios de Ciudad por Cédula Real en 1532. Los dominicos comenzaron a extender su acción evangelizadora en todo el valle. La época colonial significó el término al culto de los dioses, los templos paganos fueron destruídos y en lugar de ellos elevaron iglesias y conventos. Los misioneros trataron de desterrar ritos y costumbres, incluso los homenajes a Centéotl. Así introdujeron la fiesta de la Virgen del Carmen. En el lugar del teocalli de Centéotl se levantó una ermita (la ermita de la Santa Cruz) y en el mismo sitio los carmelitas en 1679 construyeron el templo del Carmen Alto. Esta modificación fue necesaria porque así se hizo posible la celebración de un santo religioso en las mismas fechas cuando los indígenas tenían sus ritos. ${ }^{5}$ Se verificaba la festividad el domingo siguiente al día 16 de julio (cuando no cayera el 16 en domingo) con la Procesión del Corpus y el lunes inmediato con una fiesta secularizada de los indios que repitieron ocho días después en la "Octava". De aquí viene la

\footnotetext{
${ }^{1}$ Octavio PAZ, El laberinto de la soledad, México, 1994, 51

${ }^{2}$ Guaje es una especie de acacia, el cerro abundaba en este tipo de árboles

${ }^{3}$ Centéotl -nombre masculino- era dios dual de los aztecas. En las celebraciones rendían culto al dios en su presencia de mujer (Centeocihuatl). Así existe una confusión en la interpretación actual, pues el nombre correcto utilizado en las fiestas sería Centeocihuatl. En este ensayo utilizo el nombre -actualmente conocido- de Centéotl

${ }^{4}$ En este tema véase: Gustavo Jesús PÉREZ JIMÉNEZ, Lunes del Cerro. Guelaguetza, Oaxaca, 2003, 13-14, Luis Ignacio VELÁSQUEZ, "El origen de la Guelaguetza", in: Noticias, 17 de julio de 2003, Guillermo MARÍN, La Guelaguetza, www.toltecayotl.org

5 el día de Santa Cruz es el 3 de mayo, mientras el del Virgen del Carmen es el 16 de julio

${ }^{6}$ Luis Ignacio VELÁSQUEZ, "El Lunes del Cerro durante la Colonia", in: Noticias, 19 de julio de 2003, "Guelaguetza, el arte de compartir", in: Oaxaca, julio de 2003

${ }^{7}$ La Octava es una tradición de repetir las fiestas católicas a los ocho días de haberse celebrado
} 
denominación 'Lunes del Cerro' que significa actualmente la serie de festividades -que dura casi dos semanas- cuyo momento más destacado es la Guelaguetza. Muestra claramente el proceso de la aculturación y sincretismo que desde el siglo XVII introdujeron espectáculos de España, como la "Tarasca", los "Gigantes" o las "Marmotas", los dos últimos siguen estando presentes en la actual fiesta también. Durante la época de la independencia las festividades perdieron muchas características coloniales y se convirtieron más bien en paseos al cerro. Aparecieron actividades y concursos deportivos. La gente se ponía sus mejores galas, preparaban un almuerzo que llevaban al cerro donde también tomaban ricas golosinas y nieves de frutas.

En 1932 Oaxaca celebró el IV Centenario de su elevación a ciudad. El gobierno del estado organizó en abril del año mencionado un Homenaje (o Fiesta) Racial ${ }^{9}$ en el que las siete regiones folclóricas del Estado presentaron cuadros de danza en honor de la capital pero sólo en 1942 recibe la fiesta el nombre de Guelaguetza que se incluye a Las Fiestas del Lunes del Cerro en los años $50 .{ }^{10}$

Se dice que la Guelaguetza es una derivación de una palabra zapoteca-Guendalizaá- que significa ofrenda, regalo o apoyo mutuo. Es una costumbre en comunidades indígenas oaxaqueñas dar guelaguetza (comidas, bebidas y aun dinero) en los casamientos, bautizos, fiestas, nacimientos y funerales o ayudar en las labores agrícolas como la cosecha. Las familias registran en un libro los regalos que reciben para poder corresponderlos de la misma manera. En algunas fuentes mencionan otra costumbre -ya colonial- con la misma denominación según la cual los indios solían llevar las primicias cosechadas a los hacendados españoles cuyas tierras cultivaban. La fiesta recibió este nombre debido a que las delegaciones acostumbraban a llevar productos de su región (pan, frutas, sombreros y productos artesanales) para regalarlos entre el pueblo y a los representantes del gobierno del estado e ilustres invitados. ${ }^{11}$

\section{La Guelaguetza actual}

Son las décadas de los 60,70 y 80 cuando la Guelaguetza se desarrolla y se convierte en la fiesta compleja que hoy visitan miles de turistas cada año. La serie de festividades dura unos 10 días, tienen lugar muestras gastronómicas, artesanales, actividades deportivas, representaciones teatrales y costumbristas de las diferentes comunidades del estado, conciertos y exposiciones. Los programas más importantes son los siguientes:

- Elección de la diosa Centéotl: desde 1969 cada delegación participante envía una candidata al título de "Diosa Centéotl". El sábado anterior al primer lunes se realiza un concurso donde lo que cuenta no es la belleza de las mujeres indígenas sino tratan de elegir a la mujer que más conserva las tradiciones y costumbres de su pueblo. Las participantes con sus trajes regionales hablan con orgullo en español y en su lengua nativa de sus costumbres, gastronomía, trajes, bailes o de la situación de las mujeres en su comunidad. La elegida diosa participará con las autoridades en los acontecimientos de las fiestas. En 1969 ganó la representante de la delegación de Pinotepa Nacional, este año la ganadora ha sido la representante de los Valles Centrales. ${ }^{12}$

- Desfile de delegaciones: por la tarde del mismo día los oaxaqueños salen de su casa para ver el desfile de las delegaciones que está organizado a manera de una calenda religiosa tradicional con música de banda. Aparecen los representantes y danzantes de las diferentes regiones iniciando con las chinas oaxaqueñas, los gigantes y las marmotas.

- Bani Stui Gulal: es "la repetición de la antigüedad", una representación teatral -en la Plaza de la Danza, delante de la Basílica de la Soledad- de la historia de las Fiestas de los Lunes del Cerro que se presentó por primera vez en 1969. Ponen en la escena las ceremonias antiguas de los aztecas con el

\footnotetext{
${ }^{8}$ la tarasca representaba un dragón-serpiente hecho de papel y tela; los gigantes son muñecos grandes en carrizo cubierto de papel mache representando las diferentes razas, en el baile de los gigantes participan tres parejas simbolizando una pareja indígena, una negra y otra española; las marmotas son globos grandes de carrizos cubiertos de tela blanca (en la cual en la época tratada se pintaba pasajes del Nuevo y Viejo Testamento)

${ }^{9}$ La fiesta se celebró el 25 de abril de 1932

${ }^{10}$ Gustavo Jesús PÉREZ JIMÉNEZ, op. cit., 14

${ }^{11}$ Véase: Gustavo Jesús PÉREZ JIMÉNEZ, op. cit., 15 y la página web de la Secretaría de Educación Pública: www.sep.gob.mx

${ }^{12}$ Sandra RAMOS ROJAS, "Pide Carina Coronel Vásquez apoyo hacia las comunidades", in: EL IMPARCIAL, 20 de julio de 2003, Gustavo Jesús PÉREZ JIMÉNEZ, op. cit., 52
} 
sacrificio de la representante de las diosa del maíz, la llegada de los españoles y los misioneros, el inicio de las fiestas de la Virgen del Carmen, los acontecimientos deportivos, el gozo de música y de comidas del siglo XIX y, para terminar, el espectáculo finaliza con la música, danzas y versos de las siete regiones y quema de fuegos artificiales.

- Donají, la leyenda (desde 1983): es la escenificación de una versión ${ }^{13}$ de la leyenda de la princesa Donají (su nombre significa Alma Grande), hija del rey zapoteca Cosijoeza y su esposa Coyolicatzin. La obra cuenta el nacimiento y adolescencia de la princesa, su amor con el príncipe mixteca Nucano y su muerte dolorosa. Una vez, después de un combate, el padre de Donají trajo varios prisioneros mixtecos entre los cuales había un joven que estaba casi muerto. La princesa llevó a su casa el cuerpo del herido para atenderlo y curarlo. Después de seis meses el joven Nucano (Fuego Grande) se curó y pudo regresar a su pueblo pero durante este tiempo un amor apasionado brotó entre los dos jóvenes. Según los tratados de paz concluidos entre los dos pueblos entregaron a Donají como rehén a los mixtecas y así permaneció prisionera en Monte Albán. Donají envió un mensaje a su padre para que atacara con sus tropas la fortaleza y la salvara. Mientras tanto Nucano se acercó al aposento de la princesa porque quería estrecharla entre sus brazos. Donají apenas le informó del peligro cuando algunos capitanes mixtecos entraron y salvaron al príncipe. Antes de la llegada de las tropas de Cosijoeza otros capitanes mixtecos tomaron a Donají y la decapitaron a las orillas del río Atoyac. Tiempo más tarde la cabeza apareció cerca del río con una flor de lirio que había enraizado en su sien. ${ }^{14}$ La leyenda es representada por el Ballet Folklórico de Oaxaca y es patrocinado por el Gobierno del Estado. Se lleva a cabo el lunes por la noche en el Auditorio Guelaguetza y se concluye con quema de fuegos artificiales.

La Guelaguetza ${ }^{15}$ :

El lunes y en su Octava a las 5 de la madrugada los chirimiteros ${ }^{16}$ recorriendo las calles de la ciudad se ponen a tocar las mañanitas ${ }^{17}$ y los ciudadanos salen para el cerro donde van a tomar un almuerzo oaxaqueño. Desde las ocho las Marimbas del Estado ofrecen su música a la multitud de espectadores ya en el Auditorio y a las diez da inicio la Guelaguetza ${ }^{18}$. Luego que la Diosa Centéotl recorre el escenario para después ocupar su lugar en el presidium junto a las personalidades invitadas, las delegaciones (que vienen de varios pueblos de las siete regiones -más bien a partir de la década de los noventa se empezó a hablar de ocho ${ }^{19}$ - representando 16 grupos étnicos del estado ${ }^{20}$ ) presentan una muestra de su patrimonio cultural a través de bailes, cantos, música o representación de alguna costumbre, rito o fiesta de su pueblo vistiéndose con trajes regionales. Después de cada representación los danzantes dan su guelaguetza (frutas, comidas, productos artesanales) a las personalidades invitadas y al público. En cada Guelaguetza (el primer y el segundo lunes) se cambian algunas delegaciones $^{21}$, así no se puede ver las mismas danzas en la Octava o en el transcurso de los años. Sin embargo, hay algunas que son accesorios indispensables de cada fiesta ${ }^{22}$ :

\footnotetext{
${ }^{13}$ Hay muchas versiones, la escenificada es la del padre Maximilano Amador (1927)

${ }^{14}$ Luis Ignacio VELÁSQUEZ, "Las leyendas de la Princesa Donají", in: Noticias, 01 de agosto de 2003, Gustavo Jesús PÉREZ JIMÉNEZ, op. cit., 54-55

${ }^{15}$ Véase en el tema: Gustavo Jesús PÉREZ JIMÉNEZ, op. cit., María Claudia LÓPEZ MORALES, Lunes del Cerro en la Ciudad de Oaxaca, Oaxaca, Carteles Editores 1993, Luis Ignacio VELÁSQUEZ, "La Guelaguetza, a su esplendor", in: Noticias, 22 de julio de 2003, Luis Ignacio VELẢSQUEZ, "Hoy, la octava del Lunes del Cerro", in: Noticias, 28 de julio de 2003

${ }^{16}$ la chirimía es un instrumento musical de viento traído por los españoles, parecido al clarinete, con diez agujeros y lengüeta de caña. En la actualidad significa también un conjunto musical de dos a siete personas que no solamente tocan chirimías sino tambores, flautas de carrizo y trompetas también. Las chirimías fueron incluidas en las festividades en el año de 1969.

${ }^{17}$ canción popular mexicana que se dedica a alguien el día de su cumpleaños o santo y generalmente se canta al alba

${ }^{18}$ desde los años 50 la autoridad estatal de turismo organiza las fiestas

${ }^{19}$ las ocho regiones son la de la Mixteca, la Cañada, la Sierra Norte, el Golfo, los Valles Centrales, el Istmo, la Costa y la de la Sierra Madre del Sur

${ }^{20}$ los grupos étnicos son: los amuzgos, cuicatecos, chatinos, chinantecos, chonchos, chontales, huaves, ixcatecos, mazatecos, mixes, mixtecos, nahuatlecos, triques, zapotecos, zoques y popolocas.

${ }^{21}$ Delegaciones de 2003, Primer Lunes: 1. Chinas Oaxaqueñas (Región del Valle) 2. Tamazulapan del Espíritu Santo, 3. San Melchor Betaza, 4. San Pablo Macuiltianguis (Sierrra del Norte) 5. Mihuatlán de Pofirio Díaz (Sierra del Sur) 6. Huautla de Jiménez (Cañada) 7. Juchitán de Zaragoza 8. Ciudad Ixtepec (Istmo) 9. Huajuapan
} 
Las chinas oaxaqueñas son las mujeres de los barrios de la ciudad de Oaxaca, ellas son las que venden tortillas, carne y fruta en los mercados. Son las protagonistas de las calendas religiosas. En la Guelaguetza ofrecen su calenda como bienvenida de la ciudad a los representantes de las otras regiones acompañadas por una banda de música, chirimía y los gigantes. En la cabeza llevan una canasta de flores con símbolos cristianos e interpretan el Jarabe del Valle. Sus faldas de satín brillan en varios colores que contrastan con los colores vivos de las mascadas de seda sobre los hombros. Llevan muchas joyas de oro con símbolos religiosos como el de la Virgen María.

Sones mazatecos de Huautla de Jiménez (región de la Cañada): en su forma actual fueron interpretados por primera vez en 1932. La mayor parte de los sones son versos compuestos y musicalizados en el año de 1910. Llevan huipil ${ }^{23}$ blanco bordado y adornado con listones rojos que simbolizan el café.

Entrega de Mayordomía (Tlacolula de Matamaros, región de los Valles Centrales): es la representación de una costumbre de los pueblos que tiene origen colonial. En esta época el Mayordomo costeaba los gastos necesarios para las fiestas del santo patrono. Con el transcurso del tiempo no sólo los amos españoles sino miembros de la población rural también desempeñaban este cargo. Cada año se designa un mayordomo que se celebra durante varios días con bailes y comidas. La delegación presenta esta costumbre finalizando con la interpretación del Jarabe del Valle.

Torito Serrano (San Pablo Macuiltianguis, Región de la Sierra): el baile aparece en las fiestas locales participando en él casi todos los habitantes. Es la representación de una costumbre traída por los españoles, la corrida o un juego con el toro. El papel del toro lo juega la mujer que trata de hacer lo más posible por derribar al hombre. El baile empieza con una especie de himno regional, luego viene el jarabe y más tarde el son del Torito. Los versos chuscos que acompañan al baile se dicen en zapoteco serrano.

Flor de Piña (San Juan Bautista Tuxtepex, Región del Alto Papaloapan): este baile fue creado especialmente para la Guelaguetza. Hasta el año de 1958 la región no tenía un baile que representara en las fiestas. Por este motivo el gobernador del estado envió la melodía de Flor de Piña (compuesta por un compositor oaxaqueño) a la ciudad de San Juan Bautista Tuxtepec para que crearan una coreografía que reflejara la 'tradición' de la municipalidad. Así nació el baile -actualmente uno de los favoritos del público- que hoy se interpreta únicamente por mujeres con huipiles chinantecos de colores vivos llevando una piña en el hombro (la zona es uno de los principales productores de piña del Estado) simbolizando la alegría de la mujer por la buena cosecha de esta fruta. Se dice que su original sentido ritual es el de celebrar a las deidades de la tierra y la cosecha.

La Danza de la Pluma (Cuilapan de Guerrero o Villa de Zaachila, Región de los Valles Centrales): es la primera danza institucionalizada en estas fiestas que representa la conquista de los aztecas por parte de los españoles. La música y coreografía tienen su origen en la época colonial. En la escenificación original de la conquista aparecen los principales protagonistas de ambos lados: Moctezuma, Cortés, Pedro de Alvarado y Malinche, sin embargo en la Guelaguetza sólo aparece el bando de los aztecas. Hay algunos historiadores que opinan que los movimientos ejecutados en la danza representan tácticas y movimientos guerreros o los movimientos de los astros. ${ }^{24}$ El elemento más destacado del vestuario son los altos y multicolores penachos. El danzante que personifica Moctezuma lleva un penacho tricolor, los otros portan adornos más simples.

de León (Mixteca) 10. San Juan Bautista Tuxtepec (Papaolapan) 11. Tlacolula de Matamaros 12. Cuilapan de Guerrero 13. Ejutla de Crespo (Valles Centrales) 14. Villa de Santiago Jamiltepec 15. Sta Catarina Juquila (Costa); Octava: 1. Chinas Oaxaqueñas (Región del Valle) 2. San Pedro y San Pablo Ayutla Mixe 3. Villa Hidalgo Yalálag (Sierra Norte) 4. Huautla de Jiménez (Cañada) 5. Santo Domingo Tehuantepec 6. Ciudad Ixtepec (Istmo) 7. Santiago Juxtlahuaca 8. Tlaxiaco (Mixteca) 9. Santiago Jocotepec 10. San Juan Bautista Tuxtepec (Papaolapan) 11. Ejutla de Crespo 12. Villa de Zaachila 13. San Antonio Castillo Velasco (Valles Centrales) 14. San Pedro Pochutla 15. Santos Reyes Nopala 16. Pinotepa Nacional (Costa)

${ }^{22}$ Véase en el tema de las danzas: Gustavo Jesús PÉREZ JIMÉNEZ, op. cit., Oaxaca, Tierra de Pluralidad, La Guelaguetza: Breve Semblanza, Oaxaca, Instituto Oaxaqueño de las Culturas 1993

${ }^{23}$ camisa suelta que usan las mujeres, sin mangas y con bonitos bordados

${ }^{24}$ Gustavo Jesús PÉREZ JIMÉNEZ, op. cit., 37 


\section{¿Una fiesta inventada?}

Generalmente se habla de Los Lunes del Cerro y la Guelaguetza como unas fiestas que tienen raíces y elementos prehispánicos y que evocan danzas, cantos y costumbres indígenas. Sin embargo hay muchos que ponen estas afirmaciones en duda. (Véase en las notas la cita de Mario Valdivia) ${ }^{25}$ Para poder entender el porqué de la reanimación o creación de las festividades tenemos que estudiar el contexto histórico. Durante y después de la revolución mexicana se puede observar nuevas tendencias en el pensamiento nacional. Los miembros de una corriente se exponen por la pluralidad cultural que consideran como apoyo del desarrollo social y cultural. Comienza a aumentar el interés por las herencias del pasado (indígena y colonial) que podrían ser rasgos distintivos de la nación. En la otra corriente se refleja una opinión contraria: según ellos es necesario borrar las diferencias (con el mestizaje) y crear una unidad nacional y estabilidad política. Para ellos la homogenidad de la sociedad es más importante. ${ }^{26}$ Las nuevas tendencias ganan más terreno desde los años veinte y la gobernación de Álvaro Obregón (1920-24). Según su opinión una nación moderna necesitaba una sociedad unida y estable económica y culturalmente con raíces históricas. La élite urbana empezó a explorar el campo para estudiar a la gente rural, principalmente indígena. Éste es el periodo inicial de la búsqueda de identidad común mexicana, la búsqueda de 'lo mexicano', en esta época se convierten en símbolos de identificación nacional "el charro y la china poblana bailando el jarabe tapatío"27. La renovación y modernización de la enseñanza las personificó José Vasconcelos ${ }^{28}$ (él mismo nació en Oaxaca) quien también opinó que los mexicanos necesitaban una identidad cultural unificadora. Con él comienza un redescubrimiento de las tradiciones, viejas canciones y danzas regionales. Las artes populares recobran su valor, en la música y pintura comienzan a evocar temas rurales. ${ }^{29}$ En la literatura aparecen nuevos temas: la revolución, el pasado colonial y temas indígenas. Se publican polémicas y ensayos sobre la nación, estudios del territorio nacional de México con datos de los grupos indígenas (número de indígenas, de lenguas, tradiciones y circunstancias de vida) así consiguiendo que al público también le interesen las danzas regionales, la música popular y las leyendas antiguas que más tarde serán temas de representaciones teatrales (como la leyenda de Donají). En las diferentes obras tratan de hacer resaltar los rasgos positivos de los indígenas y revaluarlos como muy mexicanos. ${ }^{30} 31$

En los años 30 -por consecuencia de la crisis económica mundial- los gobiernos tratan de estimular la producción y el comercio nacionales con "Campañas Nacionalistas", o sea una propaganda de los valores nacionales ${ }^{32}$. Principalmente durante el gobierno de Cárdenas (1934-1940) los primeros pasos

\footnotetext{
25 "El autor... no se cansará de repetir, año tras año, las denominaciones auténticas de la fiesta en la que se incluyen jarabes y danzas regionales, a lo que, para fines turísticos se llama "guelaguetza"; bello nombre para una bella costumbre indígena de proporcionar ofrendas con motivos de gratitud o simpatía en las viejas comunidades, pero que no corresponde a una fiesta del Valle de Oaxaca que tampoco tiene raíces prehispánicas, como se machaca hoy en día, habiendo caído en el fiasco histórico de hacernos creer que el espectáculo del domingo previo en la Plaza de la Danza, es una „repetición de la antigüedad”, habiendo sido en realidad una ocurrencia de un viejo cronista y recopilador de costumbres... Buen día para escuchar música típica, compuesta en su mayor parte por don Samuel Mondragón en los años veinte del siglo anterior (es falso que sean acordes prehispánicos lo que escuchamos en la guelaguetza); buen día para admirar la Danza de la Pluma, coreografía de una pieza escénica de los frailes misioneros para enseñar la religión y que se llamó 'La Conquista'." Mario de VALDIVIA, "Lunes del Cerro", in: EL IMPARCIAL, 20 de julio de 2003

${ }^{26}$ Leonel DURÁN, "Las culturas indígenas de México y su proceso de cambio de identidad", in: José ALCINA FRANCH, Indianismo e indigenismo en América, Madrid, Alianza Editorial 1990, 244

${ }^{27}$ Ricardo PÉREZ MONTFORT, Estampas de nacionalismo popular mexicano, México, Ciesas 1994, 120

${ }^{28}$ 1882-1959, en 1920 fue designado Rector de la Universidad Nacional y jefe del Departamento Universitario y de Bellas Artes; estuvo desterrado (desde 1928) de México y regresó en 1940 para ser Director de la Biblioteca Nacional

${ }^{29}$ Octavio PAZ, op. cit., 165, Historia de México, México, Salvat Mexicana de Ediciones 1986, tomo 15, 25282529

${ }^{30}$ Esta idea se extendió sobre todo entre nacionalistas izquierdistas

${ }^{31}$ Leonel DURÁN: op. cit., 245-246, Rick A. LÓPEZ, "The India Bonita Contest of 1920 and the Ethnicization of Mexican National Culture", in: The Hispanic American Historical Review, Durham, Duke University Press 2002,315

${ }^{32}$ Ricardo PÉREZ MONFORT, op. cit., 127
} 
del populismo y nacionalismo continúan y se extienden y para los años 40 llegan a su auge. ${ }^{33}$ Aparecen esfuerzos para integrar a los pueblos indígenas al proyecto de nación, para que sean miembros de la sociedad nacional y para que nazca 'lo mexicano' más o menos homogéneo. Desde los 40 y los 50 nacen cada vez más estudios etnográficos de los grupos sobrevivientes de las culturas prehispánicas. Esta reanimación folclórica inspira a que la élite blanca adopte el vestido de diferentes grupos indígenas para celebrar fiestas nacionales (el fenómeno aparece ya en los años veinte con la "moda" del vestido de los charros y chinas poblanas) y que en los años 60 comiencen a organizar concursos de belleza indígena (en toda América Latina). Este tipo de concursos existe ya en los años $20^{34}$ pero ganan más terreno en este periodo. Los organizadores subrayan que en los concursos no consideran la belleza o apariencia física sino la representación, el vestido, y las características indígenas de las concursantes. En esta época incluyen la elección de la Diosa Centéotl en las festividades de Los Lunes del Cerro (1969). ${ }^{35}$ Mientras el indigenismo significó más bien una reflexión criolla y mestiza sobre el indio, en los años 70 aparecen aspiraciones y reivindicaciones auténticamente indias, nacen organizaciones sociales indígenas (indianismo). No obstante desde estos años los indígenas también significan un recurso para el turismo y grupos étnicos que 'deben ser protegidos'.

La búsqueda de identidad no sólo aparece en el terreno estatal sino también a nivel regional. Durante los años 20 y 30 en las diferentes regiones se inicia una reactivación del patrimonio cultural y se aumenta el interés por la historia local, los ritos y costumbres. La característica más importante de Oaxaca fue su multiculturalidad desde tiempos coloniales, es la región donde mayor número de grupos étnicos hay en México. El IV Centenario de la ciudad de Oaxaca (1932) fue un buen pretexto para reanimar y para dar a conocer la música y las danzas regionales. Los indios así podían ser parte del patrimonio y las antiguas costumbres y tradiciones llegaron a ser símbolos de la ciudad. La referencia al pasado legitimó la introducción de prácticas inventadas también. Con la terminación de la carretera Panamericana (años 40) la ciudad experimenta un incremento demográfico y económico, sin embargo es -actualmente también- una de las regiones más pobres y el mayor exportador de trabajo del estado. Como consecuencia el turismo llega a ser el ingreso casi más grande de la región y por intereses ideológicos y comerciales nace la fiesta 'mixta' (en la cual la diosa que celebran y eligen es mexica, la costumbre de la Octava es religiosa, los gigantes, las marmotas y la chirimía fueron traídos por los españoles y como hemos visto muchas danzas y la mayoría de las músicas fueron creadas a lo largo del siglo XX) que llega a ser -así la mencionan- la festividad folclórica más grande de América. Con el apoyo del 'hilo' -aunque sea creado- entre el presente y el pasado, o sea con la referencia a las raíces indígenas las fiestas lograron convertirse en una tradición "antigua" y parte de la identidad de la población local ${ }^{36}$ que ofrecen y presentan al mundo y a los miles de turistas que visitan la ciudad cada año.

\section{Bibliografía}

Leonel DURÁN, "Las culturas indígenas de México y su proceso de cambio de identidad", in: José ALCINA FRANCH, Indianismo e indigenismo en América, Madrid, Alianza Editorial 1990

"Guelaguetza, el arte de compartir", Oaxaca, julio de 2003

"Guelaguetza, orgullo de Oaxaca", Directorio Turístico, Año 1, No. 2, julio de 2003

Historia de México, (coordinador general: Miguel LEÓN-PORTILLA) México, Salvat Mexicana de Ediciones 1986, tomos 6-8, 13-15.

HORVÁTH Gyula, A populizmus kialakulása Mexikóban, Kaposvár, Megyei és Városi könyvtár nyomdája 1996

"Llegan 77 mil turistas a los Lunes del Cerro", in: Noticias, 20 de julio de 2003

\footnotetext{
${ }^{33}$ Véase en el tema: HORVÁTH Gyula, A populizmus kialakulása Mexikóban, Kaposvár, Megyei és Városi Könyvtár Nyomdája 1996

${ }^{34}$ el primer concurso semejante fue el concurso de la India Bonita en 1921

${ }^{35}$ Rick A. LÓPEZ, op. cit., 306

${ }^{36}$ La consideran parte de su cultura tanto que en los años 90 los emigrantes oaxaqueños de California también empezaron a celebrar la Guelaguetza en su nuevo domicilio
} 
María Caludia LÓPEZ MORALES, Lunes del Cerro en la Ciudad de Oaxaca, Oaxaca, Carteles Editores 1993

Rick A. LÓPEZ, "The India Bonita Contest of 1920 and the Ethnicization of Mexican National Culture", in: The Hispanic American Historical Review, Durham, Duke University Press 2002

Guillermo MARIN, La Guelaguetza, www.tolteyacotl.org

Mexican Folk Dances. Sintesis de Oaxaca, página electrónica del Instituto Cultural Raíces Mexiacanas, www.folklorico.com/folk-dances/oaxaca/sintesis-oaxaca.html

Luis NAVARRO GARCÍA, Las Claves de la Colonización Española en el Nuevo Mundo 14921824, Barcelona, Editorial Planeta 1991

Oaxaca, Tierra de Pluralidad, La guelaguetza: Breve Semblanza, Oaxaca, Instituto Oaxaqueño de las Culturas 1993

Octavio PAZ, El laberinto de la soledad, México, Fondo de Cultura Económica 1994

Gustavo Jesús PÉREZ JIMÉNEZ, Lunes del Cerro. Guelaguetza, Oaxaca, 2003

Ricardo PÉREZ MONTFORT, Estampas de nacionalismo popular mexicano. Ensayos sobre cultura popular y nacionalismo, México, Ciesas, 1994

Sandra RAMOS ROJAS, "Pide Carina Coronel Vásquez apoyo hacia las comunidades", in: $E L$ IMPARCIAL, 20 de julio de 2003

Secretaría de Educación Pública (página electrónica), www.rep.gob.mx

Mario de VALDIVIA, "Lunes del cerro", in: EL IMPARCIAL, 20 de julio de 2003

Rosendo VEGA ARCOS, La magia de Oaxaca. ¿Lunes del Cerro o Guelaguetza?, Oaxaca, La Riqueza Cultural Total, Cultura para el tercer milenio A.C. 1997

Luis Ignacio VELÁSQUEZ, "El origen de la Guelaguetza", in: Noticias, 17 de julio de 2003

Luis Ignacio VELÁSQUEZ, "Cosmovisión indígena Zapoteca y Mixteca", in: Noticias, 18 de julio de 2003 2003

Luis Ignacio VELÁSQUEZ, "El Lunes del Cerro durante la Colonia”, in: Noticias, 19 de julio de

Luis Ignacio VELÁSQUEZ, "Fiesta Racial", la primera Guelaguetza oficial”, in: Noticias, 20 de julio de 2003

Luis Ignacio VELÁSQUEZ, "Los recuerdos de la fiesta racial”, in: Noticias, 21 de julio de 2003

Luis Ignacio VELÁSQUEZ, “Guelaguetza, a su esplendor”, in: Noticias, 22 de julio de 2003

Luis Ignacio VELÁSQUEZ, “¿La diosa Centeocíhuatl o el dios Centéotl?”, in: Noticias, 23 de julio de 2003

Luis Ignacio VELÁSQUEZ, "Las leyendas de la Princesa Donaji”, cinco partes, in: Noticias, 27 de julio -1 de agosto de 2003

Luis Ignacio VELÁSQUEZ, "Empieza a caer la fiesta", in: Noticias, 28 de julio de 2003

Luis Ignacio VELÁSQUEZ, "Hoy, la octava del Lunes del Cerro”, in: Noticias, 28 de julio de 2003 